\title{
The representation in memory of morphine's unconditioned motivational effects depends on the nature of the conditioned stimulus
}

\author{
TARESA L. STEFURAK \\ University of Toronto, Toronto, Ontario, Canada \\ GERARD MARTIN \\ Memorial Univeristy of Newfoundland, St. John's, Newfoundland, Canada \\ and \\ DEREK VAN DER KOOY \\ University of Toronto, Toronto, Ontario, Canada
}

\begin{abstract}
In conditioning paradigms, morphine has both rewarding and aversive motivational properties. The rewarding motivational property is preferentially associated with places, whereas the aversive property is preferentially associated with tastes. We used this preferential associability of stimuli in combination with a higher order conditioning procedure to ask how the motivational effects of morphine are represented in memory. First-order conditioning involved the pairing of a novel taste or distinct place with an intraperitoneal injection of morphine $(15 \mathrm{mg} / \mathrm{kg})$ in two separate groups of rats. As expected, conditioned taste aversions developed in one group and conditioned place preferences developed in the other. Second-order conditioning involved pairing the first-order taste conditioned stimulus (CS) with a distinct place CS in the absence of morphine in one group and pairing the first-order place CS with a distinct taste CS in the absence of morphine in the other group. If the first-order taste CS, for example, elicits from memory a representation of the entire motivational spectrum of morphine, then the second-order place cues might be expected to reveal conditioned place preferences on the basis of differential associability findings. However, if the first-order taste CS calls up only a representation of morphine's aversive effects, then second-order conditioned place aversions would be expected. We found that the firstorder taste CS produced second-order conditioned place aversions. Similarly, in the other group, the first-order place CS produced second-order taste preferences. In other words, only conditioning within one motivational system was observed. We propose that the first-order stimulus conditioned to morphine represents in memory only that single motivational effect of morphine to which the first-order conditioned stimulus is preferentially associable.
\end{abstract}

Opiates, along with other psychoactive drugs, have both rewarding and aversive motivational properties (Sherman, Pickman, Rice, Liebeskind, \& Holman, 1980; Wise, Yokel, \& Dewitt, 1976). Visual, auditory, and tactile stimuli are most readily associated with the rewarding effects of morphine, as demonstrated by the place preferences of rats for an environment distinct in these modalities that has been previously paired with morphine injections (Bechara \& van der Kooy, 1985; Mucha, van der Kooy, O'Shaughnessey, \& Bucenieks, 1982; Rossi \& Reid, 1976). In contrast, the aversive effects of morphine are preferentially associated with taste stimuli, and experimental animals avoid tastes that have been previously paired with morphine injections (Bechara \&

This research was supported by the Medical Research Council of Canada. Correspondence may be sent to Taresa Stefurak, Neurobiology Research Group, Department of Anatomy, University of Toronto, Toronto, Ontario M5S 1A8, Canada. van der Kooy, 1985; Cappell, Leblanc, \& Endreny, 1973; van der Kooy \& Phillips, 1977). The preferential associability of different stimulus modalities with the separate and opposite motivational effects of morphine presumably reflects the innate "wiring" of the rat (Bechara \& van der Kooy, 1985; Garcia \& Koelling, 1966; Garcia, McGowan, Ervin, \& Koelling, 1968). It is important to note that these conditioning effects are preferential rather than absolute. Under certain conditions, when the neural substrates where morphine acts are limited, it is possible to establish morphine-conditioned place aversions (Bechara \& van der Kooy, 1985, 1987) and morphineconditioned taste preferences (Mucha \& Herz, 1985).

We used the differential associability of place- and tasteconditioned stimuli with morphine's rewarding and aversive effects to explore the nature of the representation in memory of morphine's motivational effects. A conditioned stimulus (CS) might evoke two possible representations in memory of morphine's motivational effects. First, the 
CS may elicit a limited portion of the morphine's motivational experience. The nature of the stimulus, either place or taste, may limit the motivational effect represented in memory to only that motivational effect of morphine to which the CS is most readily associated. Thus, in secondorder conditioning, the first-order CS would have a reinforcing potential limited to only that one motivational effect. For example, taste is most readily associated with the aversive effects of morphine. If only these aversive effects were evoked when the conditioned taste was presented, then that morphine-conditioned taste would permit only further conditioned aversions to be formed in second-order conditioning schemes. Following a similar argument, if places are most readily associated with the positive motivational effects of morphine, then they would have only a rewarding potential and would produce only conditioned preferences after second-order conditioning.

On the other hand, the first-order CS may elicit a representation of the entire morphine experience-both its rewarding and aversive effects. However, only the motivational effect appropriate to the CS modality present is expressed. The preferential associability of the specific CS presented may dominate the nature of the conditioned response seen, and it may mask the existing representation of the opposing motivational effect of morphine that is not well associated with or expressed by the CS presented. The type of conditioned behavioral response seen after presenting the first-order morphine CS may not directly reflect the entire memory representation of morphine that has been associated with the first-order CS. For example, a morphine-conditioned taste may elicit a representation in memory of both the rewarding and the aversive effects of morphine, but because taste is most likely to be associated with the aversive effects, it is only the aversive properties that are expressed when a taste is used to test the representation. If this second mode of representation in memory is correct, and the presentation of a conditioned stimulus elicits the entire morphine experience, then both motivational effects of morphine would be available for association with any other secondorder cue present. A morphine-conditioned, first-order taste stimulus would then have both a rewarding and an aversive motivational potential in subsequent second-order pairings, and the expression of a rewarding or an aversive effect in second-order conditioning would depend on the modality (place or taste) of the second-order CS.

We used a higher order conditioning paradigm to discriminate between these two possible scenarios for the representation of the motivational effects of morphine in memory. In higher order conditioning, the first-order CS is first paired with an unconditional stimulus. When the first-order CS is subsequently paired with a novel secondorder CS, the primary CS itself appears to act as a reinforcer. The reinforcing power of the secondary reinforcer originates from the motivational effects attributed to the primary drug reinforcer (Crowder, Smith, Davis, Noel, \& Coussens, 1972; Kelleher, 1966; Marcus, Carnathan,
Meyer, \& Cochin, 1976; Woods \& Schuster, 1968) and, most importantly, to the way in which these motivational effects are stored in memory (Mackintosh, 1983). In the present experiments, a first-order CS (place or taste) was paired with morphine; these morphine CSs (in essence, the representations in memory of morphine's motivational effects) then were paired with a second-order taste or place CS that was, in each case, opposite to the first-order CS modality. Tests of the second-order conditioning effects provided an indirect assay of how morphine's motivational effects were represented in memory. We found that the representation of morphine's motivational effects in memory is completely dependent on the modality of the first-order CS and is limited to only the one rewarding or the one aversive effect that is preferentially associated with the specific first-order taste or place CS.

\section{METHOD}

\section{Subjects}

The subjects were adult male Wistar rats (Charles River) weighing 300-400 g. Throughout all handling and conditioning procedures, the rats were housed individually in suspended gray wire cages in a room kept at $22^{\circ} \mathrm{C}$ and lit from 0900 to $2100 \mathrm{~h}$. Purina Rat Chow was available ad lib throughout all procedures. Water was also continuously available except during training and testing phases. During these phases, intake was limited to $20 \mathrm{~min}$ a day in the experimental situation between 0900 and $1200 \mathrm{~h}$ and to $1 \mathrm{~h}$ of free access to water between 1400 and $1500 \mathrm{~h}$ each day in the home cage.

\section{First-Order Taste Conditioning}

The subjects in Group $1(n=12)$ were initially trained to consume water on a limited-access regime of $20 \mathrm{~min}$ a day for a total of 7 days. During the last 4 of these pretraining days, the rats received their water access in a gray, neutral environment box to familiarize the animals with the training apparatus to be used during second-order conditioning. During first-order taste conditioning, the rats were first presented with a novel flavored solution for $20 \mathrm{~min}$ in their home cages. Half of the group received grapeflavored Kool-aid ( $n=6$ ), the others received cherry-flavored Koolaid $(n=6)$; both flavors were unsweetened and equally preferred (Mackey, Keller, \& van der Kooy, 1986). Immediately following the flavor presentation, all subjects were injected intraperitoneally with $15 \mathrm{mg} / \mathrm{kg} / \mathrm{ml}$ morphine sulphate dissolved in physiological saline. The next day, each of these rats was presented with the other flavored solution for $20 \mathrm{~min}$ and injected with an equal volume of physiological saline vehicle. During the first-order conditioning schedule, varying numbers of pairs of morphine and saline trials were given before and between second-order conditioning trials (see below).

\section{First-Order Place Conditioning}

The subjects in Group $2(n=12)$ were pretrained to a limitedwater-access regime and also familiarized with the general place environment apparatus, including drinking water in that apparatus, exactly as described above for Group 1. On the first day of firstorder place conditioning, the rats were placed in one of two boxes, which differed in color, texture, and smell. One had black walls and a black Plexiglas floor that had been wiped with $2 \%$ glacial acetic acid solution prior to the rat's being placed inside. The second type of box had white walls and a wood chip floor that gave off a slight smell of wood (Bechara \& van der Kooy, 1985, 1989). 
The group was subdivided such that half were first placed in the black boxes $(n=6)$ and the others were placed in the white boxes $(n=6)$. Just prior to being placed in a box, each subject was injected intraperitoneally with $15 \mathrm{mg} / \mathrm{kg} / \mathrm{ml}$ morphine sulphate dissolved in saline. Each training trial lasted $20 \mathrm{~min}$. On the following day, each subject was injected with an equal volume of the saline vehicle and was placed in the alternate type of box. During the firstorder conditioning schedule, varying numbers of pairs of morphine and saline trials were given before and between second-order conditioning trials.

\section{Second-Order Conditioning}

Although the two groups differed in first-order conditioning, the procedure used in second-order conditioning was identical for both groups. Furthermore, the place environments and taste solutions were the same as those in first-order conditioning. On the first second-order conditioning day, each subject was placed in one type of box, with a flavored taste solution. For Group 1 subjects, the type of box was novel and the taste was the one previously paired with morphine during first-order conditioning. For Group 2 subjects, the taste was novel and the box was previously paired with morphine during first-order conditioning. This second-order conditioning trial lasted $20 \mathrm{~min}$; the subjects were allowed free access to the solution presented. On the following second-order conditioning day, the subjects were placed in the other box with the other taste and allowed to drink freely for $20 \mathrm{~min}$. Second-order conditioning always occurred as varying numbers of these 2-day pairs of place/taste trials. The presentations of both taste and place were counterbalanced within groups during second-order conditioning. Neither morphine nor saline was administered on second-order conditioning days.

\section{Training and Testing Procedure}

Training occurred in four 12-day cycles of first-order and secondorder conditioning trials, except for the first training set of Group 1, which occurred over 14 days. We varied the pattern and ratio of first-order to second-order conditioning trials between sets in an attempt (1) to initially establish strong first-order conditioning effects, after which second-order conditioning could be established, and (2) to control against the possible extinction of first-order conditioning that might occur during the second-order training sessions. In both groups, first-order conditioning trials were conducted first and were integrated with second-order conditioning trials within a cycle. The training schedule for Group 1 was as follows: Cycle 1, six first-order conditioning pairs were run with morphine and saline injections presented on alternative days ( 12 days) and then one second-order conditioning pair was run with each taste presented in a different place, over 2 days, giving a total of 14 days for this cycle; Cycle 2, the pattern of two first-order conditioning pairs (4 days) and then one second-order conditioning pair ( 2 days) was run twice ( 12 days for this cycle); Cycle 3, the pattern of one first-order conditioning pair ( 2 days) and then one second-order conditioning pair ( 2 days) was run three times ( 12 days for this cycle); Cycle 4 , the pattern of two first-order conditioning pairs (4 days) and then one second-order conditioning pair ( 2 days) was run twice ( 12 days for this cycle). The training schedule for Group 2 was as follows: Cycle 1, the pattern of two first-order conditioning pairs with morphine and saline injections presented on alternative days (4 days) and then one second-order conditioning pair with each place presented with a different taste, over 2 days, was run twice (12 days for this cycle); Cycle 2, the pattern of one first-order conditioning pair ( 2 days) and then one second-order conditioning pair ( 2 days) was run three times ( 12 days for this cycle); Cycle 3 , the pattern of one first-order conditioning pair ( 2 days) and then one second-order conditioning pair ( 2 days) was run three times (12 days for this cycle); Cycle 4, the pattern of one first-order conditioning pair ( 2 days) and then two second-order conditioning pairs ( 4 days) was run twice ( 12 days for this cycle). The exact ratio of pairs of first-order conditioning trials to pairs of second-order conditioning trials within a cycle is summarized on the $x$-axes in Figures 1 and 2 . After each training cycle, all subjects were tested for both taste and place conditioning. Identical test procedures were used regardless of whether first-order or second-order conditioning was being assessed. We tested place conditioning by placing each rat into a larger rectangular test box that consisted of environments identical to the conditioning boxes at each end of the test box separated by a smaller gray area (neutral zone). No flavored solutions were present in the boxes on test days. The times spent on each of the two ends of the test box were recorded over a 10 -min period. We tested taste conditioning in a two-bottle choice test by simultaneously presenting both flavors for $15 \mathrm{~min}$ in the home cage and measuring the amount of each solution consumed by each subject. Testing for first-order and second-order conditioning was done at the end of each of the four training cycles over 2 days, with secondorder conditioning effects assessed first.

\section{RESULTS}

Both first-order taste aversions and second-order place aversions were seen in Group 1 subjects. By the time of the first test period (Figure 1A), a first-order morphineconditioned taste aversion was clearly established: the subjects drank significantly less of the morphine-paired taste than the saline-paired taste $[t(11)=2.30, p<.05]$. Due to an experimenter error, no data were available from the third test period. However, an ANOVA on the data from test periods 1,2 , and 4 indicated a significant interaction of drug with test period $[F(2,22)=9.08, p<.05]$, suggesting that additional conditioning increased the strength of the expression of the morphine-conditioned taste aversion, with a strong expression of the aversion seen in the fourth test period $[t(11)=14.4, p<.05]$. Place test scores (Figure 1B) reveal that no significant second-order conditioning effects were seen in the first test period: the subjects did not spend significantly different amounts of time in either test placed $[t(11)=0.5, p>.05]$. By the fourth test period, a significant aversion to the place associated with the morphine-paired taste had developed $[t(11)=2.3, p<.05]$. The amount of time spent in the place associated with the morphine-paired taste gradually decreased as the number of second-order taste/place pairings increased, but, given that significant second-order conditioning was not seen until the fourth test period, the interaction of drug with test period did not reach statistical significance. The subjects spent less time in the place in which they had previously consumed a morphine-paired taste than in the place in which they had consumed a vehicle-paired taste after the fourth training cycle.

Group 2 subjects revealed both conditioned first-order place and second-order taste preferences. The subjects showed a strong conditioned place preference for the environment in which they had received morphine injections by the end of the third test period (Figure 2A). The amount of time spent in the morphine- versus saline-paired 
A First Order Taste Conditioning

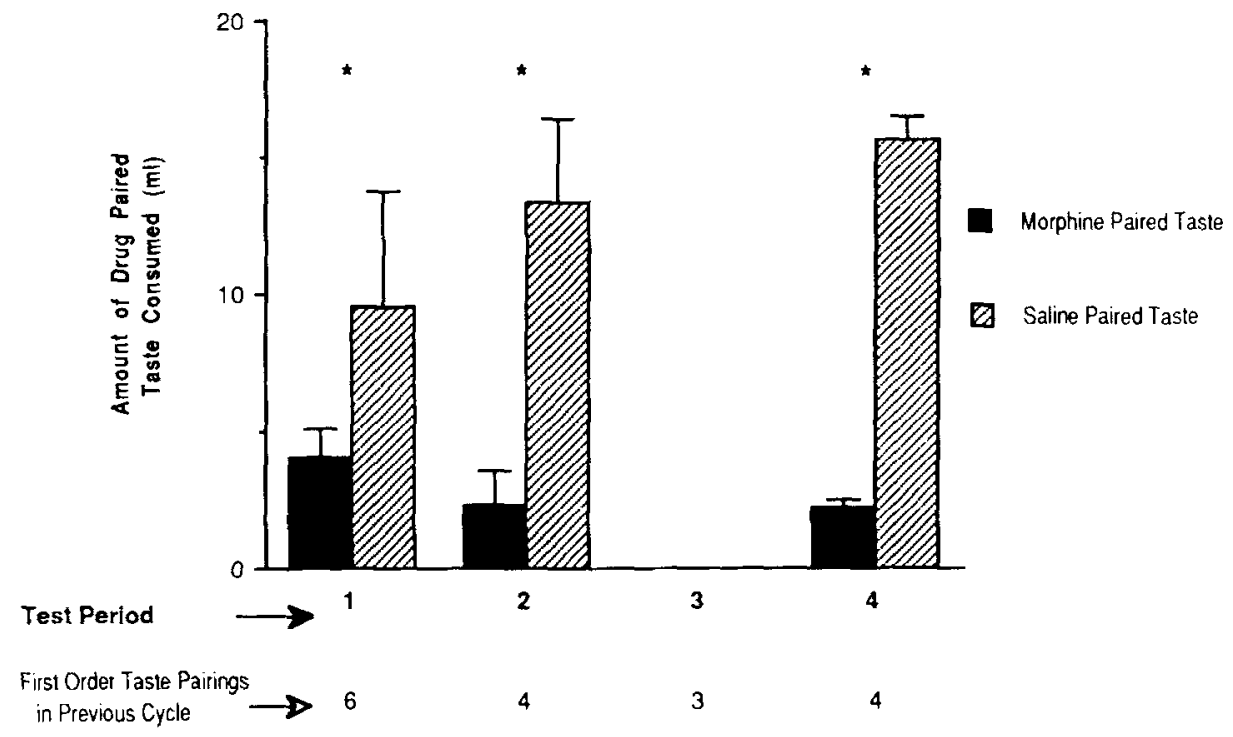

B Second Order Place Conditioning

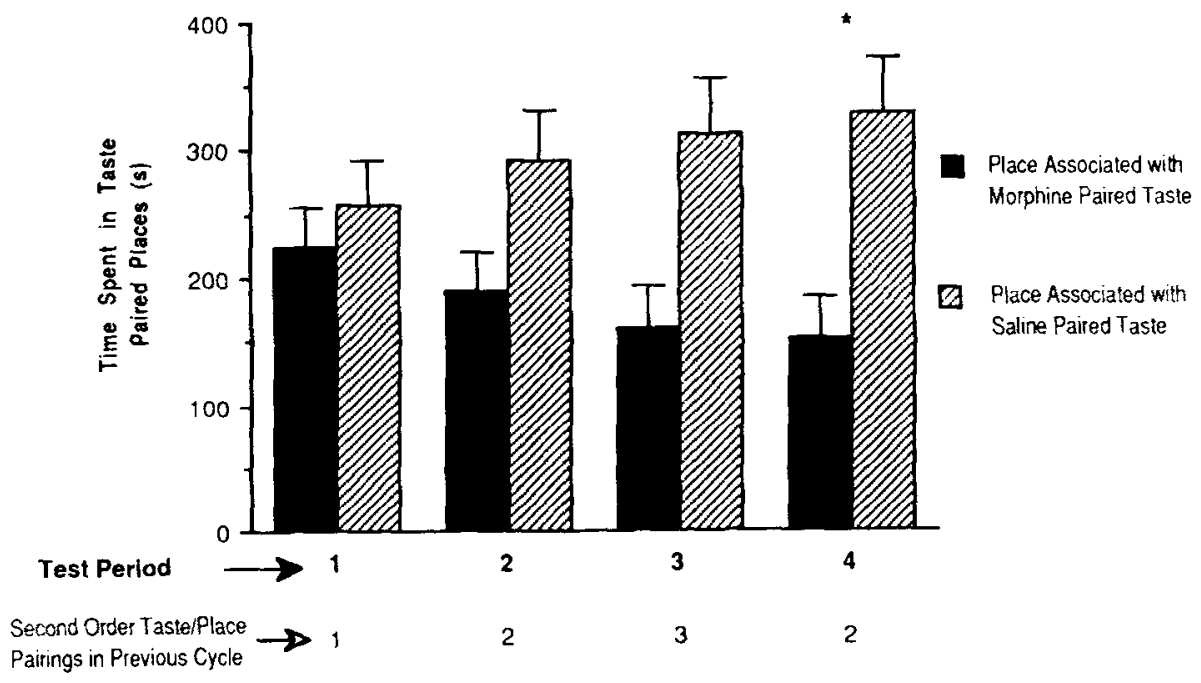

Figure 1. A: First-order conditioned taste aversions induced by morphine injection $(15 \mathrm{mg} / \mathrm{kg})$, as tested after each training cycle. Number of first-order drug/taste pairings in the cycle preceding each test period is listed along the $x$-axis. Bars represent means $\pm S E M$. Data from Test Day 3 was unavailable due to experimenter error. ${ }^{*} p<.05$, for comparison on that test day. B: Second-order conditioned place aversions induced by association of place with morphine-paired taste, as tested after each training cycle in the same animals shown in $A$. Number of second-order taste/place pairings in the cycle preceding each test period is listed on the $x$-axis. Bars represent means $\pm S E M$. ${ }^{*} p<.05$, for comparison on that test day.

places was not significantly different in the first two test periods $[t(11)=1.6, p>.05$, and $t(11)=2.08$, $p>.05$, respectively], but a significant difference was seen in both the third and fourth test periods $[t(11)=2.95$, $p<.05$, and $t(11)=4.5, p<.05$, respectively]. Indeed, an ANOVA revealed a significant interaction of drug with test period $[F(3,33)=5.27, p<.05]$. Secondorder conditioning in Group 2 (Figure 2B) revealed that, by the fourth test period, the subjects had consumed significantly more of the taste associated with the morphinepaired place $[t(11)=2.17, p<.05]$. The interaction of drug with test period in the second-order conditioning fell short of statistical significance, presumably reflecting the finding that not until the final test period was the consumption between the two tastes significantly different. The subjects drank more of the taste that they had previ- 


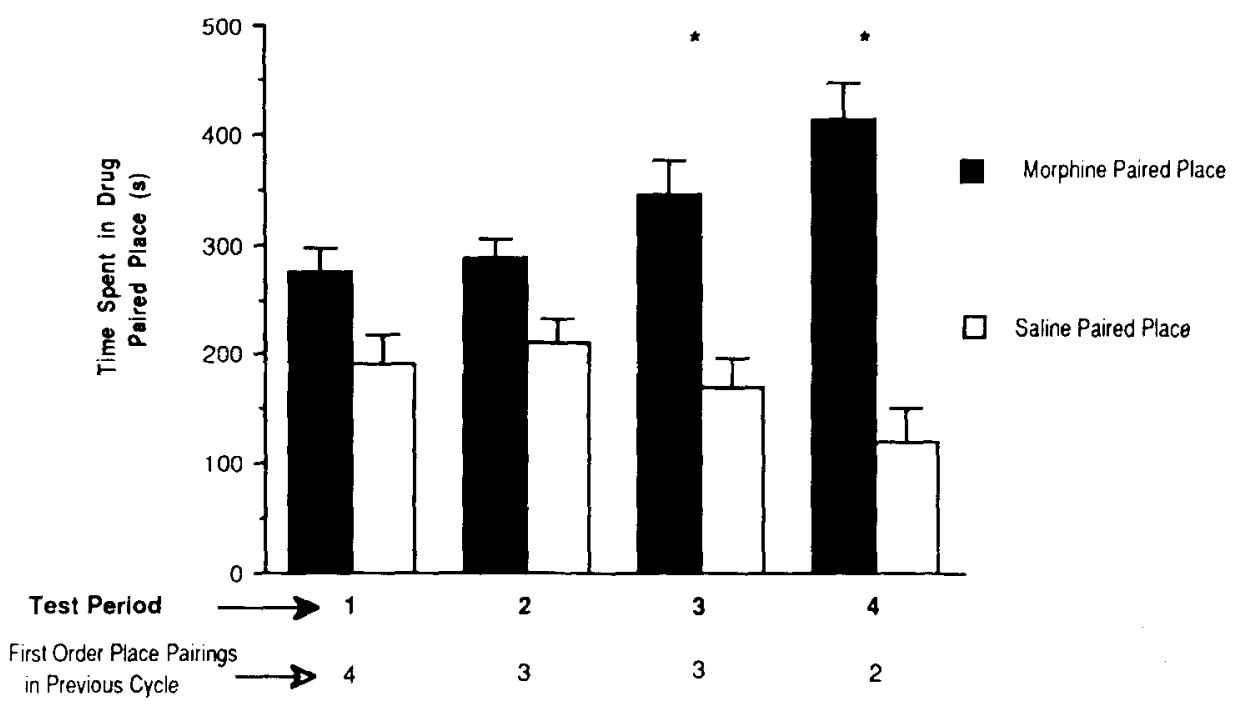

B Second Order Taste Conditioning

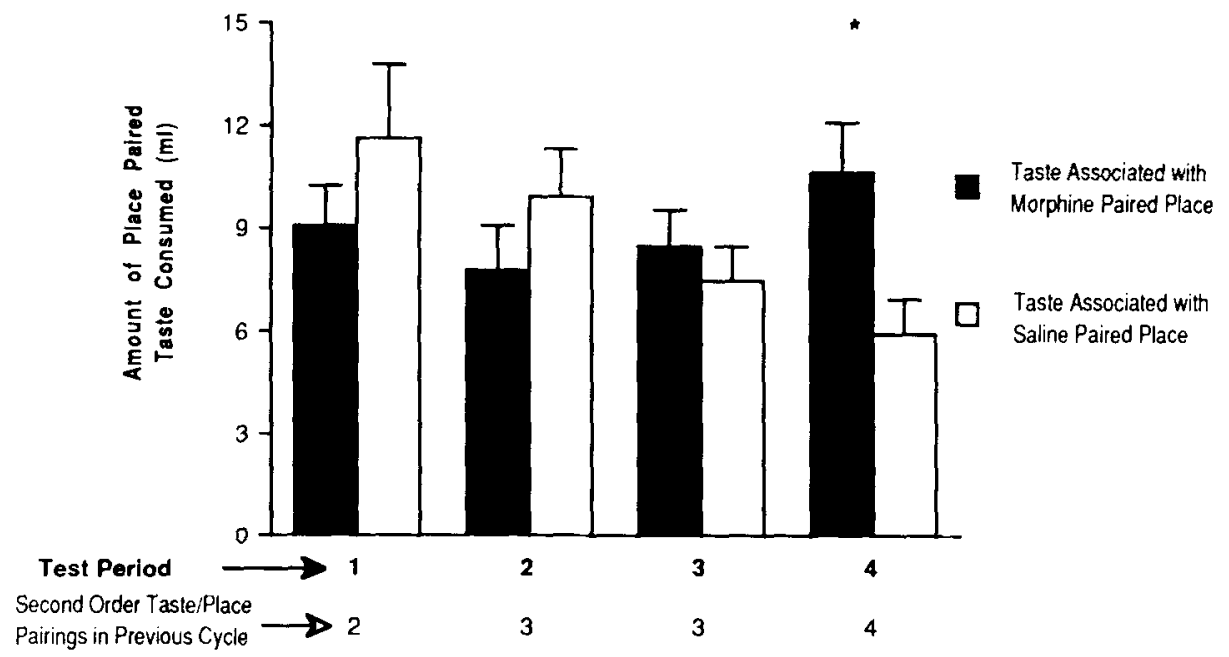

Figure 2. A: First-order conditioned place preferences induced by morphine injections $(15 \mathrm{mg} / \mathrm{kg})$. Number of first-order place/drug pairings in the cycle preceding each test period is listed along the $x$-axis. Bars represent means $\pm S E M . * p<.05$, for comparison on that test day. B: Second-order conditioned taste preferences induced by associating taste with morphine-paired place in the same animals as shown in $A$. Number of second-order place/taste pairings in the cycle preceding each test period is listed on the $x$-axis. Bars represent means $\pm S E M$. $p<.05$, for comparison on that test day.

ously consumed in a morphine-paired place than the taste that they had previously consumed in a vehicle-paired place after the fourth training cycle.

\section{DISCUSSION}

The association of a taste with morphine produced a first-order conditioned taste aversion. The pairing of this taste CS with a novel place in the absence of morphine produced second-order conditioned place aversions. This suggests that the first-order taste CS elicited only a representation in memory of morphine's aversive motivational effect. Similarly, the association of a place with morphine produced a first-order conditioned place preference. The pairing of this place CS with a novel taste in the absence of morphine induced second-order conditioned 
taste preferences. This suggests that the first-order place CS elicited a representation in memory of only morphine's rewarding motivational effect.

The first-order conditioning results confirm the previously reported (Bechara \& van der Kooy, 1985; Martin, Bechara, \& van der Kooy, 1988; Sherman et al., 1980) differential associability of tastes with the aversive motivational effect of morphine and places with the rewarding motivational property of morphine. The second-order conditioning results also show that these associations are preferential and not absolute. More importantly, the second-order conditioning results suggest that the representation of morphine's motivational effects that is evoked by a specific CS is limited to the single motivational effect that is preferentially associated with that CS during training. If a specific first-order taste or place CS had evoked a representation in memory of the entire morphine motivational experience (both the rewarding and aversive property of morphine), then the nature of secondorder conditioning presumably would have been dependent on the modality of the second-order CS. If the second-order CS was a taste cue, for example, then second-order conditioned taste aversions should have developed regardless of the modality of the first-order CS. This was not seen. The second-order conditioning effects measured were always the same (rewarding or aversive) as the first-order conditioning effects, such that only within-motivational-system second-order conditioning was observed.

The failure to see cross-motivational-system secondorder conditioning cannot be due to a limited capacity in memory for the acquisition of representations of the opposite motivational properties of morphine. Both firstorder place preferences and first-order taste aversions can be conditioned to identical injections of morphine in the same animal (Martin et al., 1988; Sherman et al., 1980). Furthermore, there appear to be no limitations in the higher order conditioning paradigm that restrict the expression of different second-order conditioning effects. The nature of the second-order conditioning response can be independent of the nature of the first-order conditioning response when the same motivational system is associated with both the first- and second-order CS (Holland, 1977; Nairne \& Rescorla, 1981). The first- and second-order CSs may elicit very different responses. Rather, the failure to see cross-motivational-system higher order conditioning in the present experiment reveals the limited representation in memory of morphine's motivational effects. This representation in memory appears to depend primarily on the modality of the primary CS. In other words, the CS with which the morphine is paired determines which subset of morphine's motivational effects becomes represented in memory.

It might be argued that both the rewarding and the aversive effects of morphine are always represented in memory but that the modality (taste or place) of the specific CS determines the type of conditioned response expressed on any one trial. It must be true that the CS determines expression because both taste aversions and place preferences can be seen after first-order conditioning in the same animal (Martin et al., 1988; Sherman et al., 1980). However, if a first-order CS presentation evokes a representation of both motivational effects of morphine from memory, then the preferential associability findings dictate that the modality of the second-order CS should determine what response is expressed during second-order conditioning. Second-order conditioning appears to operate by the same classical conditioning rules as first-order conditioning (Mackintosh, 1983), and, thus, the differential associability findings predict that the modality of the second-order CS should determine whether a second-order preference or aversion is conditioned. This was never the case in the present study. The first-order CS, and not the second-order CS, always determined whether second-order preferences or aversions were conditioned. Therefore, only one motivational effect of morphine could have been represented in memory by any one CS during first-order conditioning.

A blocking paradigm might provide one possible way to independently test the hypothesis that a CS represents only one motivational property depending on the CS modality. An association between a particular CS (CS1) and an unconditioned stimulus (UCS) can be blocked when a second CS (CS2) is introduced between CS1 and the UCS, provided that CS2 has previously acquired motivational properties through pairings with the identical UCS. Blocking occurs only within motivational systems. Blocking will not occur across motivational systems where the motivational properties of the UCS and those previously associated with CS2 are qualitatively different (Krank, 1985; Mackintosh, 1983; Parker, 1986). Using blocking to test our hypothesis, first a novel place would be paired with morphine injections such that the morphineconditioned place (CS2) is represented in memory. Second, this morphine-conditioned place would be presented as a blocking stimulus after a novel taste presentation and before a morphine injection (of the same dose as that used in the previous place association). Any UCS attenuation caused by morphine preexposure could be controlled through the inclusion of unconditioned groups with similar drug histories, and, thus, blocking effects should be detectable. We predict that if the place CS elicits a representation of only the rewarding effects of morphine (that is, only within-motivational-system storage occurs), then no blocking effects would occur between the taste and morphine association. The motivational property represented in memory by the place would be different from that property to which tastes are most readily associated, and a morphine-conditioned taste aversion would emerge. Alternatively, if the place calls up a representation of both the rewarding and aversive motivational effects of morphine, one would expect the taste/morphine association to be blocked by the morphine place CS. From the results of the present experiments, we would predict that no blocking would be seen in these experiments.

Morphine's motivational effects have been shown to be anatomically dissociable. Morphine produces its rewarding effects by acting on specific subpopulations of opiate 
receptors in the brain (Phillips \& Lepaine, 1980; Vaccarino, Bloom, \& Koob, 1985; van der Kooy, Mucha, O'Shaughnessey, \& Bucenieks, 1982). Morphine's aversive effects are produced by the action on peripheral opiate receptors, which then relay aversive information into the brain for processing (Bechara \& van der Kooy, 1985, 1987; Bechara, Zito, \& van der Kooy, 1987). The hypothesis that separate neural systems exist to process these two motivational effects of morphine is further supported by lesion evidence. Ibotenic acid and sixhydroxydopamine lesions of the visceral cortex (agranula insular) both produce a deficit that is specific to the aversive motivational properties and do not affect the rewarding effects of opiates (Mackey et al., 1986; Zito, Bechara, Greenwood, \& van der Kooy, 1988). Ibotenic acid lesions of the tegmental pedunculopontine nucleus area of the brain stem have been shown to abolish only the rewarding effects and not the aversive effects of opiates (Bechara \& van der Kooy, 1989). Lesions of either site, and the subsequent elimination of one of morphine's motivational properties, do not significantly increase the conditioned effects induced by the remaining motivational property. This suggests that the aversive and rewarding motivational properties of morphine are independent, as has been suggested by others (e.g., Faneslow, Calcagnetti, \& Helmstetter, 1989), and that the representations of these properties in memory are not integrated in the expression of conditioned effects. Therefore, we can reject the hypothesis that an integration process somehow masks one motivational property that is actually represented in memory but that cannot induce conditioning because it is integrated with a larger, dominant motivational property. Our evidence supports the hypothesis that only one of the two motivational effects of morphine is represented in memory by any one CS and is consistent with the evidence of independent and parallel neural systems mediating each of morphine's motivational effects.

It would appear then that not only are the systems for processing the rewarding and aversive motivational effects of morphine separate, but their representations in memory are also independent. Indeed, we propose that no motivational effects of morphine can be stored in memory unless supporting CSs of specific modalities are also present. Our experimental paradigm does not answer the question of what exactly is represented in memory when morphine and specific CSs are paired, but we simply suggest that some aspect of their association is represented. It has previously been shown that morphine's motivational properties are not employed as cues when morphine is used in conditional discrimination learning (Martin, Bechara, \& van der Kooy, 1987). This suggests that the motivational properties of drugs are relatively inaccessible features that impart positive or negative value to events or stimuli but are not represented by themselves in memory. It follows that for an animal to have a full representation in memory of morphine's motivational properties, both a reward-associable CS and an aversiveassociable CS need to be present during training. The al- ternate hypothesis would suggest that all of morphine's motivational effects are stored in memory with each drug presentation but that specific CSs recall only single motivational effects. Our second-order conditioning studies used different modality CSs to challenge the representation of morphine's motivational effects in memory and revealed that only single motivational effects (dependent on the nature of the initial CS) are represented in memory. This evidence concurs with previous studies showing that the processing of morphine's opposite motivational effects are independent and with suggestions that morphine's motivational effects in and of themselves are not represented in memory. The representation in memory of the motivational effects of drugs would seem to require a correlated stimulus, and, indeed, the present results suggest that the modality of the CS helps to determine the motivational valence of the representation.

\section{REFERENCES}

Bechara, A., \& VAN DER KoOY, D. (1985). Opposite motivational effects of endogenous opiods in brain and periphery. Nature, 314 , 533-534.

Bechara, A., \& VAN der Kooy, D. (1987). Kappa receptors mediate the peripheral aversive effects of opiates. Phamacology, Biochemistry \& Behavior, 28, 227-233.

Bechara, A., \& VAN der Kooy, D. (1989). The tegmental pedunculopontine nucleus: A brainstem output of the limbic system critical for the conditioned place preferences produced by morphine and amphetamine. Journal of Neuroscience, 9, 3400-3409.

Bechara, A., Zito, K., \& van der Kooy, D. (1987). Peripheral receptors mediate the aversive conditioning effects of morphine in the rat. Pharmacology, Biochemistry \& Behavior, 28, $219-225$.

Cappell, H., Leblanc, A. E., \& Endreny, L. (1973). Aversive conditioning by psychoactive drugs: Effects of morphine, alcohol and chlordiazepoxide. Psychopharmacology, 29, 239-246.

Crowder, W. F., Smith, S. G., Davis, W. M., Noel, T., * CousSENS, W. R. (1972). Effect of morphine dose size on the conditioned reinforcing potency of stimuli paired with morphine. Psychology Recordings, 22, 441-448.

Faneslow, M. S., Calcagnetti, D. J., \& Helmstetter, F. J. (1989). Modulation of appetitively and aversively motivated behavior by the kappa opioid antagonist MR2266. Behavioral Neuroscience, 103 , 663-672.

Garcia, J., \& Koelling, R. (1966). Relation of cue to consequence in avoidance learning. Psychonomic Science, 4, 123-124.

Garcia, J., McGowan, B., Ervin, F., \& Koelling, R. (1968). Cues: Their relative effectiveness as a function of the reinforcer. Science, 160, 794-795.

Holland, P. C. (1977). Conditioned stimulus as a determinant of the form of the Pavlovian conditioning response. Joumal of Experimental Psychology: Animal Behavior Processes, 3, 77-104.

KeLLEHER, R. T. (1966). Chaining and conditioned reinforcement. In W. K. Honig (Ed.), Operant behavior: Areas of research and application (pp. 160-212). New York: Appleton-Century-Crofts.

KRANK, M. D. (1985). Asymmetrical effects of Pavlovian excitatory and inhibitory aversive transfer on Pavlovian appetitive responding and acquisition. Learning \& Motivation, 16, 35-62.

Mackey, W. B., Keller, J., \& Van der Kooy, D. (1986). Visceral cortex lesions block conditioned taste aversions induced by morphine. Pharmacology, Biochemistry \& Behavior, 24, 71-78.

Mackintosh, N. J. (1983). Conditioning and associative learning. Oxford: Oxford Univeristy Press.

Marcus, R., Carnathan, G., Meyer, R. E., \& Cochin, J. (1976). Morphine-based secondary reinforcernent: Effects of different doses of naloxone. Psychopharmacology, 48, 247-250. 
Martin, G., Bechara, A., \& van der Kooy, D. (1987). Dissociation of morphine's discriminative stimulus properties from its motivational properties. Society of Neuroscience Abstracts, 3, 1546.

Martin, G., Bechara, A., \& VAN der Kooy, D. (1988). Morphine preexposure attentuates the aversive properties of opiates without af fecting the aversive properties. Pharmacology, Biochemistry \& Behavior, 39, 687-692.

Mucha, R. F., \& HeRZ, A. (1985). Motivational properties of kappa and mu opioid receptor agonists studied with place and taste conditioning. Psychopharmacology, 86, 281-285.

Mucha, R. F., van der Kooy, D., O'Shaughnessey, M., \& BuceNIEKs, P. (1982). Drug reinforcement studied by the use of place conditioning in rat. Brain Research, 243, 91-105.

NAIRNE, J. S., \& Rescorla, R. A. (1981). Second-order conditioning with diffuse auditory reinforcers in the pigeon. Learning \& Motivation, 12, 65-91.

Parker, L. A. (1986). Drug-specific blocking of lithium-, amphetamine, and apomorphine-induced conditioned flavor avoidance. Learning \& Motivation, 17, 965-968.

Phillips, A. G., \& Lepaine, F. (1980). Reinforcing effects of morphine microinjection into the ventral tegmental area. Pharmacology, Biochemistry \& Behavior, 12, 965-968.

Rossi, N. A., \& ReID, L. D. (1976). Affective states associated with morphine injections. Physiological Psychology, 4, 269-274.

Sherman, J. E., Pickman, C., Rice, A., Liebeskind, J. C., \& HolMAN, E. W. (1980). Rewarding and aversive effects of morphine; temporal and pharmacological properties. Pharmacology, Biochemistry \& Behavior, 13, 501-505.

Vaccarino, F. J., Bloom, F. E., \& KooB, G. F. (1985). Blockade of nucleus accumbens opiate receptors attenuates intravenous heroin reward in the rat. Psychopharmacology, 86, 37-42.

van der Kooy, D., Mucha, R. F., O'Shaughnessey, M., \& BuceNIEKS, P. (1982). Reinforcing effects of brain microinjections of morphine revealed by conditioned place preference. Brain Research, 243, 107-117.

van der Kooy, D., \& Phillips, A. G. (1977). Temporal analysis of naloxone attenuation of morphine-induced taste aversion. Pharmacology, Biochemistry \& Behavior, 6, 637-641.

WISE, R. A., YoKeL, R. A., \& DEWITT, H. (1976). Both positive reinforcement and conditioned aversion from amphetamine and from apomorphine in rats. Science, 191, 1273-1275.

WoOds, J. H., \& SCHUSTER, C. R. (1968). Reinforcement properties of morphine, cocaine and SPA as a function of unit dose. International Journal of Addiction, 3, 231-237.

Zito, K. A., Bechara, A., Greenwood, C., \& van der Kooy, D. (1988). The dopamine innervation of the visceral cortex mediates the aversive effects of opiates. Pharmacology, Biochemistry \& Behavior, 30, 693-699.

(Manuscript received March 5, 1990; revision accepted for publication May 30, 1990.) 